\title{
Respuesta de poblaciones microbianas que lideran el crecimiento en raíces y resistencia sistémica inducida
}

\author{
Reply of microbial populations leading the growth in roots and induced systemic resistance
}

\author{
${ }^{\bullet}$ Hayron Canchignia Martínez${ }^{1}$, María Peñafiel Jaramillo², Carlos Belezaca Pinargote ${ }^{2}$, Mercedes Carranza Patiño ${ }^{2}$ \\ Oscar Prieto Benavides ${ }^{2}$, Ramiro Gaibor Fernández ${ }^{1}$
}

${ }^{1}$ Área de Microbiología del Departamento de Biotecnología, Facultad de Ciencias Agrarias, Escuela de Agronomía, Universidad Técnica Estatal de Quevedo, Campus Ing. Manuel Haz Álvarez, km 1.5 vía a Santo Domingo de los Tsáchilas. EC.120501.Quevedo,Ecuador. ${ }^{\circ}$ hcanchignia@ute.edu.ec; rgaibor@uteq.edu.ec

${ }^{2}$ Facultad de Ciencias Ambientales, Universidad Técnica Estatal de Quevedo, Campus Ing. Manuel Haz Álvarez, km 1.5 vía a Santo Domingo de los Tsáchilas. EC.120501. Quevedo, Ecuador. mariafernandapeñafiel@gmail.com; cbelezaca@yahoo.com; mcarranza@uteq.edu.ec; oprieto@uteq.edu.ec

\section{Resumen}

L as rizobacterias lideran una serie de mecanismo en el sistema radículas en plantas que favorecen en aspectos fisiológicos como: desarrollo radicular por la producción de auxinas y promueven el mecanismo de defensa a nivel sistémico. Las rizobacterias del género Pseudomonas spp reportan tener capacidad de producir ácido indolil-3-acetico (AIA), en presencia del precursor Triptófano, bajo condiciones in vitro. La rizobacterias Promotoras de Crecimiento en Plantas (PGPR), en contacto con las raíces de la planta, estas rizobacterias utilizan los exudados de aminoácidos proteinogénico triptófano precursor para síntesis de AIA, esto incrementa la producción del fitoregulador que favorecen el desarrollo y proliferación de la biomasa radicular adventicias. Se rescata la importancia del empleo de PGPR, como efectores en el mecanismo en defensa sensu stricto para la Resistencia Sistémica Inducida (RSI), que activa el estado de prealerta en la planta antes y después de ser sometida al agente patogénico. La RSI se activa por la ruta dependiente al jasmonato (JA) y etileno (ET).

Palabras clave: rizobacterias, triptófano, agente patógeno, mecanismo de defensa

Recibido: 30-noviembre-2014. Recibido en forma corregida: 25-febrero-2015. Aceptado: 27-marzo-2015.

Publicado como ARTÍCULO DE REVISIÓN en Ciencia y Tecnología 8(2): 1-11 Diciembre de 2015

\begin{abstract}
$\mathrm{R}$ hizobacteria are an alternative that has proven not to generate resistance in pathogens. Pseudomonas strains play an important role in biocontrol, because they provide a great variety of bioactive compounds to control plant pathogens. The information search focused on the study of Pseudomonas spp isolates which have the ability to diminish the viability of such pathogens as: fungi, bacteria, nematodes through the use of an antagonist mechanism inducing plant defense systems through systemic acquired resistance (SAR) and induced systemic resistance (ISR) activating the pre-alert state on the ground before and after being subjected to the pathogenic agent. The RSI for the dependent route to jasmonate (JA) and ethylene (ET) is activated.
\end{abstract}

Key words: rhizobacteria, tryptophan, pathogen defense mechanism 


\section{Introducción}

$\mathrm{D}$ iferentes rizobacterias que promueven el crecimiento en las plantas (PGPR), dirigen la mayoría de los controles biológicos de hongos patogénicos como: Phytophthora spp, Fusarium spp, Verticillium spp, estos afectan las raíces de plantas. Las rizobacterias se encuentran en la rizósfera y de forma indirecta favorecen al desarrollo radicular y supervivencia de la planta al patógeno (Sacherer et al., 1994; Heeb and Haas, 2001).

Las PGPR inducen el desarrollo de raíces al estar en contacto con ellas. Woodward y Bartel (2005), indican que el ácido indolil-3-acético (AIA) es la principal auxina en plantas, al controlar muchos procesos fisiológicos: 1) división y alargamiento celular, 2) diferenciación de tejidos. Kravchenko et al. (2004), manifiestan que la síntesis de AIA por la bacteria en asociación con la planta, captan el triptófano del producto de degradación en raíces y los exudados radiculares. Las rizobacterias mejoran la proliferación de raíces por la producción de AIA (Asghar et al., 2004; Khalid et al., 2004).

Las PGPR están ejerciendo un efecto sinérgico hacia la planta, contribuyendo a su crecimiento (Preston, 2004) y ofreciendo un efecto de imprimación (Van Loon et al., 1998), que ofrece una activación rápida y fuerte de las respuestas de defensas antes y después de ser expuestas a un patógeno. Se ha descrito el rol de P. fluorescens como agente controlador de patógenos fúngicos (Dwivedi y Johri, 2003), donde ellas están ejerciendo un control del patógeno por un efecto antagonista a través de la síntesis de compuestos con actividad antifúngica.

PGPR se pueden encontrar entre los diferentes géneros de bacterias: Arthrobacter, Chryseobacterium, Curtobacterium, Gluconacetobacter, Azospirillum, Azotobacter, Bacillus, Burkholderia, Herbaspirillum, y Pseudomonas. La Resistencia Sistémica Inducida (RSI) en Arabidopsis thaliana por efecto de la aplicación de la cepa Bacillus sp L81 y Arthrobacter oxidans BB1, se obtiene una disminución en el índice de la enfermedad de 61.20 y $52.30 \%$ respectivamente (Barriuso et al., 2008).

El empleo o presencia de $P$. fluorescens, ha demostrado ser un activador de RSI en varias especies de plantas, que protegen sistemáticamente contra diferentes patógenos de hongos que atacan la raíz, por Fusarium oxysporum (Léon et al., 2005). Rizobacterias no patógenas de $P$. fluorescens CHA0, P. fluorescens WCS417 y $P$. aeruginosa 7NSK2 reducen las enfermedades en los tejidos a través de la inducción de una defensa conocida como RSI, en Vitis vinifera contra Botrytis cinirea (Verhagen et al., 2009).

La información disponible del trabajo, está enfocado a la aplicación de rizobacterias que promueven el crecimiento por producción de fitorreguladores y la inducción de los genes de resistencias para promover la RSI por las vías de señalización dependientes al etileno y jasmonato en plantas.

\section{Rizobacterias productoras de auxinas y el estímulo al desarrollo radicular en plantas}

PGPR productoras de auxinas. La producción de auxinas AIA, ha sido verificada en condiciones in vitro al inocular las PGPR en medios de cultivos selectivos para la obtención de este fitorregulador. Oberhänsli et al. (1991) obtuvieron una producción mínima de AIA $0.02 \mathrm{mM}$ a las $72 \mathrm{~h}$, en presencia de triptófano $(10 \mathrm{mM})$ por P. fluorescens CHA0. A diferencia de Sridevi y Mallaiah (2007) observaron un aumento en producción de AIA (de 0.074 a $0.159 \mathrm{mM}$ ) al emplear triptófano (de 1.50 a $2.50 \mathrm{mg} \mathrm{mL}^{-1}$ ) a $72 \mathrm{~h}$ de muestreo en Rhizobium spp. Se puede deducir que las rizobacterias tienen diferente respuesta de producción de AIA in vitro, bajo la presencia de triptófano y tiempo de muestreo.

Las bacterias con mayores concentraciones de AIA se encuentran: Azospirillum brasilense (20-40 $\mu \mathrm{g} /$ $\mathrm{mL})$, Azospirillum paspali $\left(35 \mu \mathrm{g} \mathrm{mL}^{-1}\right)$, P. fluorescens J-143 (14.60 $\mu \mathrm{g} \mathrm{mL}^{-1}$ ) (Mantilla, 2007; Hernández et al., 2004). Otros microorganismo reportan menos producción de AIA con $(0.09 \mathrm{mM})$ por Paenibacillus polymyxa KNUC6, que incrementa la masa radicular en Capsicum annuum (pimienta), pero reduce la infección del patógeno Erwinia carotovora (Phi et al., 2010), de igual manera Teixeira et al. (2007), realiza aplicaciones de Bacillus subtilis (3918) que secretan $\left(0.70 \mathrm{ug} \mathrm{mL}^{-1}\right)$ de AIA, incrementando el enraizamiento en $6.80 \%$ en mini-estacas de E. grandis y E. urophylla.

PGPR promotoras en el desarrollo de raíces. Las rizobacterias del suelo tienen la capacidad de colonizar raíces y estimular el crecimiento en plantas. Esta capacidad de promover el crecimiento ha sido relacionada con diferentes actividades fisiológicas: 1) La síntesis de fitohormonas, como son citoquininas, giberelinas y auxinas; 2) Mejora los factores que afectan la nutrición mineral, como la solubilización de fosforo; 3) Protección de plantas hacia fitopatógenos (Rodríguez et al., 2007).

La aplicación de rizobacterias del género Rhizobium spp, aisladas de nódulos de raíces de Sesbania sesban (sesbania), estimulan el crecimiento de raíz por la producción de fitohormonas de AIA (Sridevi y Mallaiah, 2007). El AIA es una fitohormona que estimula el crecimiento vegetal, por incremento de biomasa, altura de tallos, crecimiento del vástago principal (dominancia apical) y reduce el crecimiento 
de ramas laterales (Koshiba, 1993).

Taiz y Zeiger (2006) manifiestan que el triptófano está presente en las raíces. Una vez que la bacteria entra en contacto con el tejido radicular, ella capta pequeñas cantidades de triptófano presente en los tejidos, que darían paso a la producción de AIA y favoreciendo así el aumento de pelos absorbentes y raicillas. Bialek et al. (1992) indican que las plantas sintetizan auxinas naturales de AIA y es sintetizada a partir del L-triptófano, que se encuentra libre en la planta o formando parte de proteínas. Ljung et al. (2001), manifiestan que los tejidos de las plantas son capaces de sintetizar auxinas, la mayoría se produce en los ápices meristemáticos de tejido verde (tallos, primordios de hoja y hojas jóvenes).

Se corrobora que las auxinas incrementan el desarrollo de raíces adventicias, López et al. (2009) observan un incremento de pelos radiculares en $A$. thaliana y una respuesta normal de pelos radiculares en plantas mutantes de Arabidopsis que carecen de los genes aux1-7 y eir1-1 al emplear Bacillus megaterium. Esto coincide con Barka et al. (2000) al realizar aplicaciones de Pseudomonas sp. PsJN en plantas in vitro de Vitis vinifera L. cv 'Chardonnay' incrementan el desarrollo pelos absorbentes. De una manera Glick et al. (1999), explican que las bacterias que secretan bajos niveles de AIA (10-9 a 10-12 M), estimulan la elongación de raíces, mientras que bacterias altamente productoras de auxinas promueven la formación de raíces laterales o el desarrollo de pelos absorbentes.

Las PGPR a más de ejercer el control biológico hacia agentes patógenos, favorece el incremento de pelos absorbentes de raíces principales en plantas, que benefician la absorción de los nutrientes minerales que se encuentran en la superficie del suelo. Lo que realza la importancia en el rescate y selección de nuevos individuos de PGPR, para ser empleados en campo y beneficiar el desarrollo de la planta y actúen como bio-controlador. La aplicación de P. fluorescens CHA0 como agente bio-controlador, se encuentra restringido, por la patente que puntualiza el uso del antibiótico a la función específica de la síntesis del 2,4 DAPG, limitando su empleo para el control de agentes patogénicos (Thomashow et al., 1996).

\section{Efectores bacterianos para activar la resistencia sistémica inducida}

Las bacterias poseen determinantes para estimular la RSI como: componentes de envoltura celular lipopolisacáridos (LPS), metabolitos secretados, antibióticos, componentes reguladores de hierro (sideróforo). Los LPS de P. fluorescens WCS417, están implicados en liderar la RSI contra Pseudomonas syringae pv. en tomate (Yan et al., 2002) y A. thaliana (Van Wees et al., 1997).
Lipopolisacáridos. Los LPS de P. fluorescens WCS374 y WCS417 inducen la resistencia sistémica en Raphanus sativus (rábano) hacia el hongo patógeno $F$. oxysporum., esto fue verificado en cepas mutantes de $P$. fluorescens WCS417, que carece de la cadena lateral del antígeno-O de los LPS, observando como resultado la inactivación en la inducción de la RSI. Indicando que la cadena lateral del antígeno-O de los LPS, sirve como una señal a liderar en la inducción del mecanismo de defensa en esta planta (Leeman et al., 1996).

Caso contrario en otros estudios, los LPS de P. fluorescens WCS417r y mutantes de WCS417r que carecen de la cadena lateral del antígeno-O de LPS, estos llegan a regular el mecanismo de defensa en A. thaliana (Van Wees et al., 1997). Se puede definir que la RSI está regulada por LPS presentes en PGPR y que este mecanismo de activación para RSI difiere en dependencia de la planta hospedera. Los lipopolisacáridos no solo es la única característica en la determinación de RSI. Los LPS de P. fluorescens WCS374 Y WCS417 son los mayores determinantes de RSI bajo condiciones repletas de hierro, caso contrario bajo condiciones limitantes de hierro, los LPS de estas bacterias no están implicadas en RSI en rábano (Cuadro 1) citado por Van Wees et al. (1997).

Sideróforos. Los sideróforos producidos por Pseudomonas spp (pseudobactina o pioverdina), son moléculas de bajo peso molecular secretadas por los microorganismos para captar hierro desde el medio-ambiente este modelo de acción le confiere un mecanismo de supresión de enfermedades, basándose en competencia por hierro con el patógeno. Además, interesantemente los sideróforos pueden inducir el sistema de resistencia RSI (Bakker et al., 2003). Las pseudomonas, pueden suprimir cepas patogénicas de F. oxysporum por competición mediada por sideróforos para hierro férrico (Leeman et al., 1995).

La pseudobactina producido por estas bacterias, es responsable de la RSI, la aplicación de purificados de pseudobactina, aislado desde P. fluorescens WCS 374, y su aplicación a raíces de rábano induce la RSI (Ramamoorthy et al., 2002).

Ácido salicílico. Distintas Pseudomonas spp producen un sideróforo no fluorescente denominado pyochelin, de un péptido cysteinyl substituido por salicílico (Cox et al., 1981). El ácido salicílico es producido por Pseudomonas spp, bajo una limitación de hierro. El AS es otro metabolíto cuyo papel fundamental está relacionado con la inducción de RSI (Leeman et al., 1995). Bacterias que pierden la capacidad de producir AS, también pierden su capacidad de producir resistencia en plantas (De Meyer y Hofte, 1997). 
Cuadro 1. Efectores bacterianos para RSI por Pseudomonas spp en diferentes plantas

\begin{tabular}{lll}
\hline Determinantes & Pseudomonas & Planta hospedera \\
\hline Componentes de envoltura celular & & \\
Flagelo & P. putida WCS358 & Arabidopsis \\
Lipopolisacaridos & P. fluorescens WCS374 & Rábano \\
& P. fluorescens WCS417 & Arabidopsis \\
& P. putida WCS358 & Arabidopsis y tomate
\end{tabular}

Metabolitos reguladores de hierro

Derivados de benzilamino N-alquilados

Sideróforo pseudobactina

Acido salicílico

Antibióticos

2,4- diacetilfloroglucinol

$\begin{array}{ll}\text { P. putida } \text { BTPI } & \text { Frijol } \\ \text { P. fluorescens } \text { CHA0 } & \text { Tabaco } \\ \text { P. fluorescens } \text { WCS374 } & \text { Rábano } \\ \text { P. putida } \text { WCS358 } & \text { Tomate }\end{array}$

$\begin{array}{ll}\text { P. aeruginosa } 7 \mathrm{NSK} 2 & \text { Frijol } \\ \text { P. aeruginosa } 7 \mathrm{NSK} 2 & \text { Tabaco } \\ \text { P. fluorescens } \mathrm{P} 3 \text { pchBA } & \text { Tabaco }\end{array}$

P. fluorescens CHA0 Arabidopsis y tomate

P. fluorescens Q2-87 Arabidopsis
Antibióticos. El antibiótico 2,4-DAPG, además de ser un anti-fúngico, lidera la RSI en $A$. thaliana, este antibiótico es producido por $P$. fluorescens CHA0, es el compuesto clave en la resistencia contra el hongo Perospora parasítica (Iavicoli et al., 2003). La aplicación de la bacteria P. fluorescens Q2-87 y el compuesto puro de 2,4-DAPG, lidera la RSI en plantas de $A$. thaliana y Solanum liycopersicum (tomate) frente $P$. syringae. Esto fue comprobado al emplearse bacterias mutantes que no producen este antibiótico y no inicia el mecanismo de defensa por RSI (Weller et al., 2012). En tomate, la aplicación de $P$. fluorescens $\mathrm{CHA} 0$ induce la resistencia sistémica frente al nematodo $M$. javanica. Se ha demostrado al trabajar con bacterias mutantes al antibiótico 2,4-DAPG son ineficientes a liderar este mecanismo de respuesta de RSI (Siddiqui y Shoukat, 2003).

Activación de la resistencia sistémica en Arabidopsis thaliana y su mecanismo de defensa a patógenos

Activación del mecanismo de defensa por RSA (AS) y RSI (JA/ET). El patógeno al estar en contacto con el tejido de la planta, pone en marcha un sistema de comunicación molecular entre ambos, que desencadena la inducción de mecanismo de defensa en la planta (Desender et al., 2007). La señalización de RSA, por la transformación de Nicotina tabacum (tabaco) que expresan de forma constitutiva el gen bacteriano $\mathrm{NahG}$, que codifica la enzima salicilato hidroxilasa que convierte el AS en catecol, que impide la acumulación del AS. Demostrando que la acumulación de AS es requerido para la expresión de proteínas relacionada a patógenos PRs que activarían la SAR (Van Loon, 1997). Similarmente en genotipos de $A$. thaliana incapaces de sintetizar AS por plantas mutantes NahG (deficiente en inducción de ácido salicílico), esta acumulación de AS es activado por la vía de transducción-señal dependiente a RSA, que requiere de la funcionalidad del factor regulación de proteínas a patógenos (NPR1) (Figura 1).

Las proteínas PR se sintetizan y se acumulan en los tejidos de plantas en presencia de patógenos ocasionados por: infecciones por virus, bacterias y hongos, o por heridas asociadas al ataque por depredadores (Nimchuk et al., 2003). En caso particulares la presencia del hongo patogénico Plasmopara vitícola, estimula la expresión de genes de proteínas PR, (PR2; PR4; PR5; PR10) y los genes (PAL; CHS; CHI; LDOX) en plantas resistentes "Gloire de Montpellier" (Vitis riparia) (Kortekamp, 2006). V. vinifera, cv. Chardonnay, expuesta a Botrytis cinérea y Plasmopara vitícola, que incrementa la expresión de genes de defensa y de señalización como un mecanismo de respuesta a patógenos (VvNHL1; VvEDS1; PAL; 9LOX; HSR1; STS) (Chong et al., 2007). 

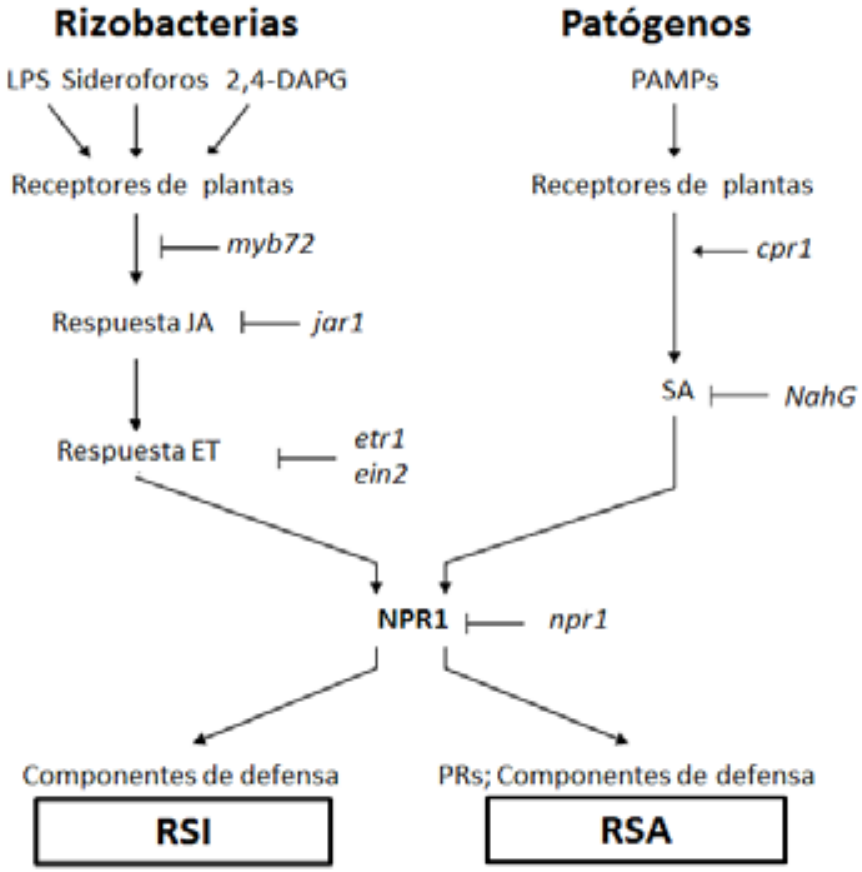

Figura 1. Representación esquemática, de las vías de transducción de señal, para la RSI mediada por rizobacterias y RSA inducida por patógeno en A. thaliana. Las flechas indican estimulación, las barras $(T)$ indican represión. PAMPs: pratrones moleculares asociados-patogenos, LPS: lipolisacaridos, JA: ácido jasmonico, ET: etileno, AS: ácido salicílico, PRs: proteínas relacionadas a patógenos. Adaptado desde el modelo de Doornbos et al. (2012)
Activación del mecanismo de defensa por RSI (JA/ ET). La activación del mecanismo de defensa por bacterias no patogénicas fue investigado por Verhagen et al. (2004), quienes manifestaron que la RSI proporciona a la planta mayor capacidad en responder rápida y eficientemente a una infección ocasionada por agentes patógenos como: hongos, bacterias o virus, y sólo se activaría tras su contacto. La colonización de raíz de $A$. thaliana por $P$. fluorescens WCS417r estimula la RSI dependiente al jasmonato/etileno. Se ha demostrado que el gen MYB72 es sensible y se ve activado en el sistema radicular al estar en contacto con $P$. fluoresccens WCS417r, que estarían codificando la proteína R2R3MYB como factor de transcripción, componentes esenciales durante el primer paso de la cascada de señalización en $A$. thaliana (Van der Ent et al., 2008).

El gen MYB72 es un miembro de una amplia familia de genes R2R3-MYB, de los cuales 125 genes han sido descritos para A. thaliana (Yanhui et al., 2006). Los factores de transcripción R2R3-MYB están implicados en la regulación de varios procesos en plantas, aunque la función de la mayoría de ellas aún se desconoce (Stracke et al., 2001). Existen pocos estudios realizados a demostrar la activación de los genes de defensa para iniciar con el proceso de imprimación para la RSI en cultivares agrícolas de interés. Frente a esta temática, varios autores proceden a la búsqueda de información de genes de defensa involucrados en la respuesta de RSI en A. thaliana.

Los estudios realizados por Van der Ent et al. (2008), llegaron a demostrar la inactivación de la RSI por P. fluoresccens WCS417r, al trabajar con plantas mutantes de $A$. thaliana al (knockout del T-DNA de los genes myb72-1 y myb72-2), siendo incapaces de mantener la RSI a patógenos: Pseudomonas syringae, Hyaloperonospora parasítica, Alternaria brassicicola y Botrytis cinérea. Además, la sobre-expresión del gen MYB72 no mejora la resistencia hacia los patógenos. Las aplicaciones exógenas de etileno en A. thaliana, induce los niveles del gen myb72-1. Esto confirma que el regulador transcripcional MYB72, actúa como un nuevo componente de señalización de RSI, que es requerido en las raíces durante los primeros pasos de señalización rizobacterias-RSI (Figura 2) (Van der Ent et al., 2008). 


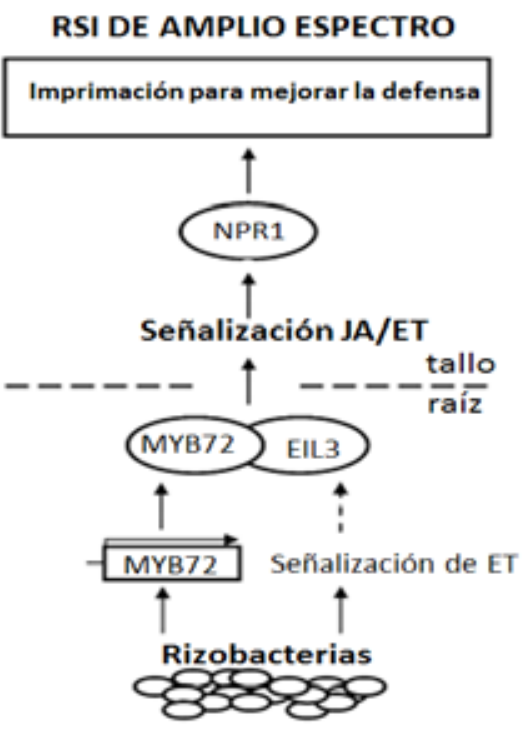

Figura 2. Modelo propuesto para el rol de MYB72 en las vías de transducción de señal controlando la RSI mediada por rizobacteria

Características y funciones de los genes relacionados a defensa. Este trabajo se ha direccionado a la recopilación de algunos de los genes que participan en los mecanismos de defensa a nivel sistémico: EL mecanismo de respuesta sistémica convergen con el gen Nprl que es dependiente de la ruta de AS. Los trabajos realizados por Van der Ent et al. (2009), manifestaron que la colonización en raíces de $A$. thaliana por $P$. fluorescens WCS417r dirigen la RSI, activando un factor de transcripción MYB72 adquiriendo una cascada de transducción de señales que convergen con NPR1. El gen nprl es el factor regulador que participa en la activación de los genes en defensa PRs; El gen eirl es el que sirve como transportador de flujo de auxinas específico para raíces, funciona como una proteína de unión a membrana localizada exclusivamente en

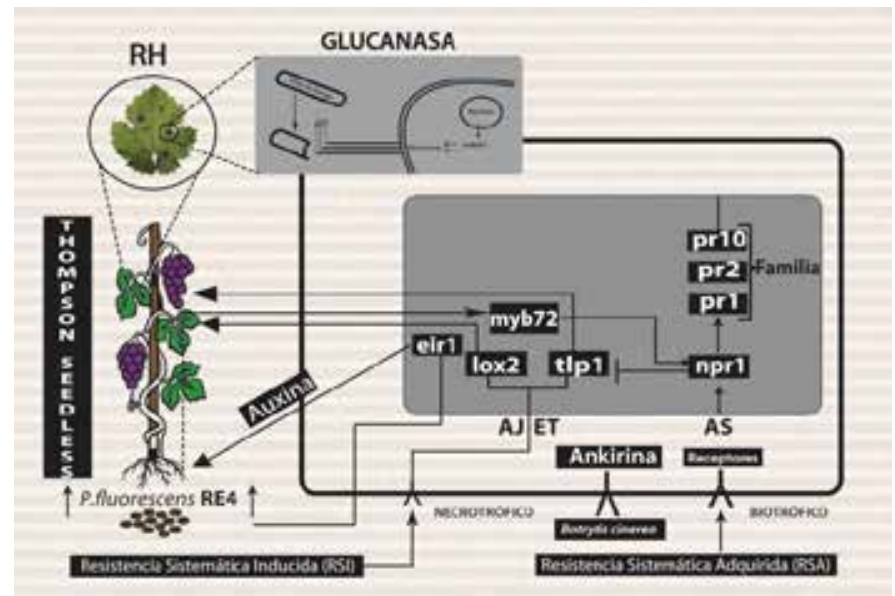

Figura 3. Representación esquemática para RSI en plantas de 'Thompson Seedless' de raíces colonizadas con $P$. fluorescens. Las flechas indican estimulación, las barras $T$ indican represión. Se ilustra la expresión de los genes relacionados a defensa por la vía del AJ y ET que dan paso a la RSI. La inoculación de $P$. fluorescens en raíces de 'Thompson Seedless' promueve el estímulo del gen Lox 2 en hojas y Tlp1, Eir1 en raíces 
Cuadro 2. Información correspondiente a genes: lox2, Eir1, npr1, Tlp1, Ankirina

\begin{tabular}{|c|c|c|c|c|}
\hline Genes & Tratamiento de inducción & Planta hospedera & Análisis & Referencia \\
\hline \multirow{4}{*}{ Lox2 } & P. fluorescens WCS417r & Arabidopsis thaliana & $\begin{array}{l}\text { ARN Blot, en hojas muestreadas a 1y } 2 \\
\text { días. }\end{array}$ & $\begin{array}{l}\text { Van Wees et al., } \\
1999\end{array}$ \\
\hline & $\begin{array}{l}\text { Pseudomonas syringae pv } \\
\text { tomato DC } 3000 \text { y Metil } \\
\text { jasmonato (MeJa) }\end{array}$ & $\begin{array}{l}\text { Arabidopsis thaliana, } \\
\text { incapaces de acumular AS } \\
\text { (Nahg) }\end{array}$ & $\begin{array}{l}\text { ARN Blot, en hojas muestreadas a } 1 \text { y } 2 \\
\text { días. }\end{array}$ & Spoel et al ., 2003 \\
\hline & P. fluorescens WCS417r & Arabidopsis thaliana & $\begin{array}{l}\text { Northern Blot, en hojas muestreadas a } 1 \text {, } \\
3 \text { y } 6 \text { horas. }\end{array}$ & Pozo et al ., 2008 \\
\hline & $\begin{array}{l}\text { Piriformospora indica } \\
\text { (hongo) }\end{array}$ & Arabidopsis thaliana & $\begin{array}{l}\text { RT-PCR cuantitativa, en hojas } \\
\text { muestreadas a } 3 \text { días. }\end{array}$ & Stein et al., 2008 \\
\hline \multirow{3}{*}{ Eir1 } & P. fluorescens CHA0r & $\begin{array}{l}\text { Arabidopsis thaliana, } \\
\text { mutantes eir } 1-1\end{array}$ & $\begin{array}{l}\text { Esporulación del hongo Peronospora } \\
\text { parasítica, en hojas muestreadas a } 5 \\
\text { días. (silvestres, eir1-1). }\end{array}$ & Iavicoli et al ., 2003 \\
\hline & Ácido naftalenacético (ANA) & Arabidopsis thaliana & $\begin{array}{l}\text { Gel de proteína, para PIN2, de plantas } \\
\text { tratadas con ANA, a } 30 \mathrm{~min} \text {. }\end{array}$ & Pan et al., 2009 \\
\hline & AIA & $\begin{array}{l}\text { Arabidopsis thaliana } y \\
\text { mutantes eir } 1-1\end{array}$ & $\begin{array}{l}\text { Q-PCR, expresión del gen pin2 a } 30 \text { y } \\
60 \mathrm{~min} .\end{array}$ & $\begin{array}{l}\text { Effendi y Scherer, } \\
2011\end{array}$ \\
\hline \multirow{3}{*}{$N p r 1$} & $\begin{array}{l}\text { P. fluorescens WCS417r; } \\
\text { MeJa; 1-aminociclopropano-1- } \\
\text { ácido carboxílico (ACC) }\end{array}$ & $\begin{array}{l}\text { Arabidopsis thaliana } y \\
\text { mutantes } n \text { pr } 1\end{array}$ & $\begin{array}{l}\text { Índice de enfermedad (\%) y RT-PCR } \\
\text { para pr-1, en hojas tratadas y } \\
\text { muestreadas a } 3 \text { días. }\end{array}$ & $\begin{array}{l}\text { Pieterse et al ., } \\
1998\end{array}$ \\
\hline & $\begin{array}{l}\text { Pseudomonas putida } \\
\text { LSW17S; Meja; AS; } P \text {. } \\
\text { syringae pv tomate }\end{array}$ & $\begin{array}{l}\text { Arabidopsis thaliana } y \\
\text { mutantes } n \text { pr } 1\end{array}$ & $\begin{array}{l}\text { Índice de enfermedad (\%) y RT-PCR } \\
\text { para pr-1, en hojas tratadas y } \\
\text { muestreadas a } 5 \text { días. }\end{array}$ & Ahn et al ., 2007 \\
\hline & $\begin{array}{l}\text { P. fluorescens WCS417r; } \\
\text { benzothiadiazol (BTH), hongo } \\
\text { Hyaloperonospora parasítica }\end{array}$ & $\begin{array}{l}\text { Arabidopsis thaliana } y \\
\text { mutantes } n \text { pr } 1\end{array}$ & $\begin{array}{l}\text { Grado de penetración del hongo (\%). } \\
\text { Mejorando la deposición de callosa, } \\
\text { atenuando la penetración. }\end{array}$ & $\begin{array}{l}\text { Van der Ent et al., } \\
2008\end{array}$ \\
\hline \multirow{3}{*}{ Tlp1 } & P. fluorescens $\mathrm{Pf} 1$ & $\begin{array}{l}\text { Lycopersicon esculentum } \\
\text { Mill. Tomate }\end{array}$ & $\begin{array}{l}\text { Por Western blot, para isoforma TLP, de } \\
\text { raíces tratadas. }\end{array}$ & $\begin{array}{l}\text { Ramamoorthy et } \\
\text { al., } 2002\end{array}$ \\
\hline & $\begin{array}{l}\text { P. fluorescens WCS417r, en } \\
\text { presencia de } P \text {. syringae pv. }\end{array}$ & $\begin{array}{l}\text { Arabidopsis thaliana Ler- } \\
0 \text { y mutantes SGT5141 } \\
(\text { AtTLPl) }\end{array}$ & $\begin{array}{l}\text { Reducción de la enfermedad a la } \\
\text { repuesta de RSI y RSA, en hojas } \\
\text { muestreadas a } 4 \text { días. }\end{array}$ & León et al., 2005 \\
\hline & $\begin{array}{l}\text { Xanthomonas oryzae pv. } \\
\text { oryzae }\end{array}$ & Arroz cv. IR 50. & $\begin{array}{l}\text { Por Western blot, inducción de la TLP, } \\
\text { en muestras de hojas, a } 1 \text { a } 6 \text { días. }\end{array}$ & $\begin{array}{l}\text { Velazhahan et al., } \\
2007\end{array}$ \\
\hline Ankirina & $\begin{array}{l}\text { Ácido salicílico (AS), } \\
\text { Pseudomonas syringae } \\
\text { virulenta }\end{array}$ & Arabidopsis thaliana & $\begin{array}{l}\text { Por RNA blot de hojas, muestreadas a } \\
\text { los } 20 \text { días }\end{array}$ & Lu et al ., 2003 \\
\hline
\end{tabular}

el tejido radicular; El estímulo del gen Lox2 da como resultado la producción de lipoxigenasa que lleva a la biosíntesis de JA; El gen Tlpl pertenece a la familia de PR-5 de proteínas relacionadas a patógenos PR, con actividad antifúngica; El gen Ankirina es un regulador de metabolismo anti-oxidantes confiriendo resistencia a enfermedades y respuesta a estrés. Nosotros ponemos en consideración el modelo de activación del mecanismo de defensa para activación de RSI y RSA en vides (Figura 3).

En este trabajo destacamos las funciones de los
TLPs, es que participan en la actividad anti-fúngica y actúan como permeabilizantes de membranas fúngicas (Batalia et al., 1996). El gen AtTPL1 codifica una proteína conocida como taumatina, que pertenece a la clase evolutivamente conservada de las proteínas PR-5 (Van Loon y Van Strien, 1999). La mayoría de las proteínas de tipo PR-5 poseen actividad antimicrobiana demostrada en ensayos in vitro, inhibiendo la germinación y provocando la lisis de las esporas de hongos y Oomycetes patógenos (Abad et al. 1996). En A. thaliana las proteínas Ankirina mayormente están involucradas en la regulación de 
metabolismo anti-oxidación confiriendo resistencia a enfermedades y respuesta a estrés (Yan et al., 2002). La proteína transmenbrana BDA1 con los repetidos dominios de Ankirina reclutarían los componentes de señalización para activar la síntesis de AS y la expresión génica de WRKY70, regulando el sistema inmune en A. thaliana (Yang et al., 2012). La función de LOXs en plantas afecta la viabilidad de las células y regula la muerte celular durante la respuesta $\mathrm{RH}$, un mecanismo de defensa a patógenos biotróficos (Rustérucci et al., 1999). Se recopiló información de genes de defensa vinculados al mecanismo de activación en plantas por RSA y RSI, que pueden ser estudiados para verificar la activación de la resistencia sistémica (Cuadro 2).

\section{Conclusiones}

U na de las nuevas alternativas para el control biológico de patógenos, está siendo liderada por la aplicación de rizobacterias no patogénicas. Existen suelos endémicos con una microfauna que favorecen al desarrollo de plantas agrícolas y mantener su producción constante sin la adición de fertilizantes químicos. Estos suelos no han sufrido cambios en su estructura biológica donde ellas están contribuyendo al control de patógenos por un efecto antagonista a los agentes patogénicos, siendo controlados por los metabolitos secundarios y la síntesis de compuestos anti-fúngicos. Además, estos metabolitos secundarios lideran el mecanismo de defensa para la RSI, que varían su activación por especie vegetal. Las rizobacterias ejercen un efecto sinérgico hacia la planta, contribuyendo al desarrollo radicular por la producción de reguladores de crecimiento de tipo auxinico y brindando un efecto de imprimación, que ofrece una activación rápida y fuerte de las respuestas de defensas antes y después de ser sometidas a un patógeno.

\section{Bibliografía}

Abad, L., Paino-Durzo, M., Lin, D., Narasimhan, L., Renveni, M., Zhu, J., Niu, X, Singh, N., Hasegawa, P., Bressan, R. 1996. Antifungal activity of tobacco osmotin has specificity and involves plasma membrane permeabilization. Plant Science 118: 11-23.

Ahn, P., Lee, S., Suh, S. 2007. Rhizobacteria-Induced Priming in Arabidopsis is dependent on Ethylene, Jasmonic Acid, and NPR1. 20: 759-768.

Asghar, H., Zahir, Z., Arshad, M. 2004. Screening rhizobacteria for improving the growth, yield and oil content of canola (Brassica napus L.).
Australian Journal Agricultural Research 55: 187194.

Bakker, P., Ran, L., Pieterse, C., Van Loon, L. 2003. Understanding the involvement of rhizobacteria mediated induction of systemic resistance in biocontrol of plant diseases. Section of Phytopathology 25: 5-9.

Barka, E., Belarbi, A., Hachet, C., Nowak, J., Audran, J. 2000. Enhancement of in vitro growth and resistance to gray mould of Vitis vinifera co-cultured with plant growth-promoting rhizobacteria. FEMS Microbiology Letters 186: 91-95.

Barriuso, J., Ramos, B., Gutiérrez, F. 2008. Protection Against Pathogen and Salt Stress by Four Plant Growth-Promoting Rhizobacteria Isolated from Pinus sp. on Arabidopsis thaliana. The American Phytopathologicla Society 98: 6

Batalia, M., Monzingo, A., Ernst, S., Roberts, W. Robertus, J. 1996. The crystal structure of the antifungal protein zeamatin, a member of the thaumatin-like, PR-5 protein family. Natural Structural Biology 3: 19-23.

Bialek, K., Michalczuk, L., Cohen, J. 1992. Auxin biosynthesi during seed germination in Phaseolus vulgaris. Plant Physiology 100: 509-517

Chong, J., Henanff, G., Bertsch, C., Bernard, W. 2007. Identification, expression analysis and characterization of defense and signaling genes in Vitis vinifera. Plant Physiology and Biochemistry 46: 469-481.

Cox, D., Rinehart, L., Moore, L., CooK, J. 1981. Pyochelin: Novel structure of an iron-chelating growth promoter of Pseudomonas aeruginosa. Proceedings of the National Academy of Sciences 78: 4256-4260.

De Meyer, G., Hofte, M. 1997. Salicylic Acid Produced by the Rhizobacterium Pseudomonas aeruginosa 7NSK2 Induces Resistance to leaf infection by Botrytis cinerea on Bean. The American Phytopathological Society 87: 588-593.

Desender, S., Andrivon, D., Val, F. 2007. Activation of defence reactions in Solanaceae: where is the specificity. Cellular Microbiology 9: 21-30.

Doornbos, R., Van Loon, L., Bakker, P. 2012. Impact of root exudates and plant defense signaling on bacterial communities in the rhizosphere. Agronomy for Sustainable Development 32: 227 243.

Dwivedi, D., Johri, B. 2003. Antifungal from fluorescent pseudomonads: Biosynthesis and regulation. Currente Science 85: 1693-1703.

Effendi, Y., Scherer, G. 2011. Auxin Binding-Protein1 (ABP1), a receptor to regulate auxin transport 
and early auxin genes in an interlocking system with PIN proteins and the receptor TIR1. Plant Signaling \& Behavior 6: 1-3.

Glick, B., Patten, C., Holguin, G., Penrose, D. 1999. Biochemical and genetic mechanisms used by plant growth promoting bacteria. Imperial College press. London. 270 p.

Heeb, S., Haas, D. 2001. Regulatory roles of the GacS/ GacA two-component system in plant-associated and other Gram negative bacteria. Molecular Plant-Microbe Interactions 14: 1351-1363.

Iavicoli, A., Boutet, E., Buchala, A., Metraux, J. 2003. Induced systemic resistance in Arabidopsis thaliana in response to root inoculation with Pseudomonas fluorescens CHA0. Molecular Plant Microbe Interactions 16: 851-8.

Khalid, A., Arshad, M., Zhair, Z. 2004. Screening plant growth-promoting rhizobacteria for improving growth and yield of wheat. Journal Applied Microbiology 96: 473-480.

Koshiba, M., Matsuyama, H. 1993. An in vitro system of indole-3-acetic acid formation from tryptophan in maize (Zea mays) coleoptile extracts. Plant Physiology 102: 1319-1324.

Kravchenko, L., Azarova, T., Makarova, M., Tikhonovich, I. 2004. Theeffect of tryptophan present in plant root exudates on the phytostimulating activity of rhizobacteria. Microbiology 73: 156-158.

Leeman, M., Den Ouden, F., Van Pelt, J., Dirkx, F., Steijl, H., Bakker, P., Schippers, B. 1996. Iron availability affects induction of systemic resistance against Fusarium wilt of radish by Pseudomonas fluorescens. Phytopathology 86: 149-155.

Leeman, M., Van Pelt, J., Den Ouden, F., Heinsbroek, M., Bakker, M., Schippers, B. 1995. Induction of systemic resistance against Fusarium wilt of radish by lipopolysaccharides of Pseudomonas fluorescens. Phytopathology 85: 1021-1027.

Léon, K., Verhagen, B., Keurentjes, J., VanPelt, J., Rep, M, Van Loon. L., Pieterse, C. 2005. Colonization of the Arabidopsis rhizosphere by fluorescent Pseudomonas spp. activates a root-specific, ethylene-responsive PR-5 gene in the vascular bundle. Plant Molecular Biology 57: 731-748.

Ljung, K., Rishikesh, P., Bhalerao, D., Sandberg, G. 2001. Sites and homeostatic control of auxin biosynthesis in Arabidopsis during vegetative growth. The plant journal 28: 465-474.

López, B., Campos, J., Valencia, E., Velázquez, C., Farías, R., Macías, L. 2009. Bacillus megaterium modifica la arquitectura de la raíz de Arabidopsis independientemente de auxinas y etileno. Biológicas. 11: 01-08.
Lu, H., Rate, D., Song, J., Greenberg, J. 2003. ACD6, a Novel Ankyrin Protein, is a regulator and an effector of salicylic acid signaling in the Arabidopsis defense response. The Plant Cell. 15: 2408-2420.

Luschnig, C., Gaxiola, R., Grisafi, P., Fink, G. 1998. EIR1, a root-specific protein involved in auxin transport, is required for gravi-tropism in Arabidopsis thaliana. Genes Development 12: 2175-2187.

Mantilla, E. 2007. Evaluación de la acción de un bioinoculante sobre un cultivo de crisantemo (Chrysanthemun morifolium var. Yoko ono) en periodo de enraizamiento. Tesis de grado. Microbiólogo agrícola y veterinario. Pontificia Universidad Javeriana. Bogota. 127 pp.

Nimchuk, Z., Eulgem, T., Holt, B., Dangl, J. 2003. Recognition and response in the plant immune system. Annual Review Genetics 37: 579-609.

Oberhänsli, T., Défago, G., Haas, D. 1991. Indole3 -acetic acid (IAA) synthesis in the biocontrol strain CHAO of Pseudomonas fluorescens: role of tryptophan side chain oxidase. Journal of General Microbiology 137: 2273-2279.

Pan, J., Fujioka, S., Peng, J., Chen, J., Li, J., Chen, R. 2009. The E3 ubiquitin ligase SCFTIR1/AFB and membrane sterols play key roles in auxin regulation of endocytosis, recycling, and plasma membrane accumulation of the auxin efflux transporter PIN2 in Arabidopsis thaliana. The Plant Cell 21: 568580.

Phi, Q., Yu-Mi, P., Keyung-Jo, S., Choong-Min, R., Seung-Hwan, P., Jong-Guk, K., Sa-Youl, G. 2010. Assessment of Root-Associated Paenibacillus polymyxa groups on growth promotion and induced systemic resistance in pepper. Journal of Microbiology and Biotechnology 12: 1605-1613.

Pieterse, C., Van Wees, S., Van Pelt, J., Knoester, M., Laan, R., Gerrits, H., Weisbeek, P., Van Loon, P. 1998. A novel signaling pathway controlling induced systemic resistance in Arabidopsis. The Plant Cell 10: 1571-1580.

Pozo, M., Van Der Ent, S., Van Loon, L., Pieterse, C. 2008. Transcription factor MYC2 is involved in priming for enhanced defense during rhizobacteriainduced systemic resistance in Arabidopsis thaliana. New Phytologist 180: 511-523.

Preston, M. 2004. Plant perceptions of plant growthpromoting Pseudomonas. Philosophical Transaction of the Royal Society 359: 907-918.

Ramamoorthy, V., Raguchander, T., Samiyappan, R. 2002. Induction of defense-related proteins in tomato roots treated with Pseudomonas fluorescens Pf1 and Fusarium oxysporum f. sp. Lycopersici. 
Plant and Soil 239: 55-68.

Rodríguez, D., Sardanelli, M., Ruíz, J. 2007. Attachment of bacteria to the roots of higher plants. FEMS Microbiology Letters 272: 127-136.

Rustérucci, C., Montillet, J., Agnel, J., Battesti, C., Alonso, B., Knoll, A., Bessoule, J., Etienne, P., Suty, L., Blein, J. 1999. Involvement of lipoxygenase-dependent production of fatty acid hydroperoxides in the development of the hypersensitive cell death induced by cryptogein of tobacco leaves. The Journal of Biological Chemistry 274: 36446-36455.

Sacherer, P., Défago, G., Haas, D. 1994. Extracellular protease and phospholipase $\mathrm{C}$ are controlled by the global regulator gene gacA in the biocontrol strain Pseudomonas fluorescens CHA0. FEMS Microbiology Lett 116: 155-160.

Siddiqui, I., Shaukat, S. 2003. Suppression of rootknot disease by Pseudomonas fluorescens CHA0 in tomato: importance of bacterial secondary metabolite, 2,4-diacetylphloroglucinol. Soil Biology and Biochemistry 35: 1615-1623.

Spoel, S., Koornneef, A., Claessens, S., Korzelius, J., Van Pelt, J., Mueller, M., Buchala, A., Métraux, J., Brown, J., Kazan, K., Van Loon, L., Dong, X., Pieterse, C. 2003. NPR1 modulates crosstalk between salicylate and jasmonate dependent defense pathways through a novel function in the cytosol. The Plant Cell 15: 760-770.

Sridevi, M., Mallaiah, K. 2007. Bioproduction of indole acetic acid by Rhizobium strains isolated from root nodules of green manure crop, Sesbania sesban (L.) Merr. Iranian Journal of Biotechnology 5: 178-182.

Stein, E., Molitor, A., Kogel, K., Waller, F. 2008. Systemic resistance in Arabidopsis conferred by the mycorrhizal fungus Piriformospora indica requires jasmonic acid signaling and the cytoplasmic function of NPR1. Plant Cell Physiologi 49: 1747-1751.

Stracke, R., Werber, M., Weisshaar, B. 2001. The R2R3-MYB gene family in Arabidopsis thaliana. Current Opinion in Plant Biology 4: 447-456

Suzuki, S., Yuxi, H., Oyaizu, H. 2003. Indole-3-acetic acid production in Pseudomonas fluorescens HP72 and its association with suppression of creeping bentgrass brown patch. Current Microbiology 47: 138-143.

Taiz, L., Zeiger, E. 2006. Auxin: The growth hormone. En: Plant Physiology. Sinauer Associates. Inc. Publishers (4 Ed). Sunderland, Massachusetts, USA. 467-507.

Teixeira, D., Couto, A., Gonçalves, R., Ferreira, E., Siqueira, L., Maffia, L., Mounteer, A. 2007.
Rhizobacterial promotion of Eucalypt rooting and growth. Brazilian Journal of Microbiology 38: 118-123.

Thomashow, L. 1996. Biological control of plant root pathogens. Current Opinion in Biotechnology 7:343-347.

Van der Ent, S., Verhagen, B., Van Doorn, R., Bakker, D., Verlaan, M., Pel, M., Proveniers, L., Van Loon, J., Pieterse, C. 2008. MYB72 is required in early signaling steps of rhizobacteria-induced systemic resistance in Arabidopsis. Plant Physiology 146: 1293-1304.

Van der Ent, S., Van Hulten, M., Pozo, M., Czechowski, T., Udvardi, M., Pieterse, C., Ton, J. 2009. Priming of plant innate immunity by rhizobacteria and $\beta$-aminobutyric acid: differences and similarities in regulation. New Phytologist 183: 419-431.

Van Loon, L. 1997. Induced resistance and the role of pathogenesis related proteins. European Journal Plant Pathology 103:753-765

Van Loon, L., Van Strien, E. 1999. The families of pathogenesis-related proteins, their activities, and comparative analysis of PR-1 type proteins. Physiological and Molecular Plant Pathology 55: 85-97.

Van Loon, L., Bakker, A., Pieterse, C. 1998. Systemic resistance induced by rhizosphere bacteria. Annual Review Phytopathology 36: 453-483.

Van Wees, S., Luijendijk, M., Smoorenburg, I., van Loon, L., Pieterse, C. 1999. Rhizobacteriamediated induced systemic resistance (ISR) inArabidopsis is not associated with a direct effect on expression of known defense-related genes but stimulates the expression of the jasmonateinducible gene Atvspupon challenge. Plant Molecular Biology. 41: 537-549.

Velazhahan, R., Jayaraj, J., Khabbaz, S., Kumar, S., Muthukrishnan, S. 2005. Xanthomonas oryzae pv. oryzae infection triggers accumulation of phenolics, defense-related enzymes and thaumatin-like proteins in rice leaves. Archives of Phytopathology and Plant Protection 39: 329-339.

Verhagen, B., Glazebrook, J., Zhu, T., Chang, H., van Loon, L., Pieterse, C. 2004. The transcriptome of rhizobacteria-induced systemic resistance in Arabidopsis. Molecular Plant Microbe Interactions 17: 895-908.

Verhagen, B., Trotel, P., Couderchet, M., Hofte, M. and Aziz, A. 2009. Pseudomonas spp. induced systemic resistance to Botrytis cinerea is associated with induction and priming of defence responses in grapevine. Journal of Experimental Botany 12: $1-12$.

Weller, D., Van Pelt, J., Mavrodi, D., Pieterse, 
C., Bakker, P., Van Loon, L. 2012. Induced systemic resistance (ISR) in Arabidopsis against Pseudomonas syringae pv. tomato by 2,4-diacety lphloroglucinol(DAPG) producing Pseudomonas fluorescens. Phytopathology 4: 403-412.

Woodward, A., Bartel, B. 2005. Auxin: Regulation, Action, and Interaction. Annals of Botany 95: 707735.

Yan, J., Wang, J., Zhang, H. 2002. An ankyrin repeatcontainingprotein plays a role in both disease resistance and antioxidation metabolism. Plant Journal 29: 193-202.

Yang, Y., Zhang, Y., Ding, P., Johnson, K., Li, X., Zhang, Y. 2012. The Ankyrin-Repeat transmembrane protein BDA1 functions downstream of the Receptor-Like Protein SNC2 to regulate plant immunity. American Society of Plant Biologists 159: 1857-1865.
Yanhui, C., Xiaoyuan, Y., Kun, H., Meihua, L., Jigang, L., Zhaofeng, G., Zhiqiang, L., Yunfei, Z., Xiaoxiao, W., Xiaoming, Q. 2006. The MYB transcription factor superfamily of Arabidopsis: expression analysis and phylogenetic comparison with the rice MYB family. Plant Molecular Biology 60: 107-124 